\title{
L'entrée de la Faculté à l'hôpital. Reflets
}

Par Jean de Blonay

A l'occasion de ce centième anniversaire, il nous a paru intéressant de retrouver l'impact qu'a eu la naissance de la faculté, tant sur l'hôpital que sur la population, les malades, les médecins, les étudiants et, surtout, sur les sciences médicales. Malheureusement, les documents sont étonnamment rares, notamment ceux qui pourraient nous donner des indications sur les faits quotidiens et banaux. Voici néanmoins quelques reflets des répercussions de l'entrée de la faculté à l'hôpital.

Le 23 octobre 1876, les auditeurs quittent l'aula de l'Université où ils viennent d'assister à l'inauguration officielle de la Faculté de Médecine. Malgré le sérieux que leur impose le cérémonial académique, certains d'entre eux dissimulent mal leur joie.

Gustave Julliard savoure le fruit de ses efforts. La Faculté fut son idée à lui. Il lui était en effet apparu inimaginable que Genève, ville académique aux institutions d'enseignement réputées, que Genève, dotée d'un hôpital perfectionné et d'un corps médical excellent, que Genève n'ouvre pas la première faculté de médecine de la Suisse Romande.

Son ami Carl Vogt s'était passionné pour le projet et avait efficacement plaidé sa cause devant Antoine Carteret, alors président du département de l'instruction publique $^{1}$. Carteret était un président dynamique, soucieux du maintien et de la promotion de son département. Son adhésion ne fut pas difficile à emporter.

Depuis lors, la question avait été débattue plusieurs fois au Grand Conseil comme nous venons de le voir grâce à l'exposé de Roger Joris. Ces discussions aboutirent à la loi du 13 septembre 1873, transformant l'Académie de Genève en Université et créant en son sein la Faculté de Médecine.

Trois ans s'étaient écoulés depuis cette mémorable séance du 13 septembre. Trois années qui avaient semblé bien longues à Gustave Julliard, mais qui avaient été nécessaires pour éviter l'improvisation et s'assurer d'un bon fonctionnement dès le départ.

A la porte de l'Université, le cortège s'organise. Inquiets, les participants scrutent le ciel. La bise modérée qui soufflait ce matin et annonçait le beau temps, a tourné en un vent d'Ouest chargé de nuages. Pourvu qu'il ne pleuve pas sur les hauts de forme et les jaquettes! Sans être excessif, le froid surprend un peu après la chaleur de l'aula. Il fait à peine dix degrés. Sous l'œil des badauds de Genève, toujours friands de ce genre de spectacle, le cortège s'ébranle enfin. 
Julliard, premier doyen de la faculté et professeur de clinique et policlinique chirurgicale, Julliard domine de sa haute taille. Il se retourne brièvement vers ses collègues dont la nomination a été entérinée le 18 février. Une belle et impressionnante équipe ${ }^{2}$.

C'est d'abord son ami Jaques-Louis Reverdin, revenu de Paris, couvert de gloire par ses travaux sur les greffes non pédiculées et l'uréthrotomie interne. Chirurgien-adjoint du service ordinaire, il enseignera la pathologie externe, la médecine opératoire et la chirurgie militaire. A eux deux, ils se partagent l'enseignement chirurgical.

L'enseignement médical sera l'affaire de Léon Revillod pour la clinique et la policlinique de médecine, de Pierre-Louis Dunant pour l'hygiène, d'Adolphe d'Espine qui créera plus tard la chaire de pédiatrie, mais qui débutera avec la pathologie interne et un cours facultatif mais fort fréquenté d'auscultation et de percussion. Jean-Louis Prévost, médecin-chef du service ordinaire, enseignera la thérapeutique et la pharmacologie, deux branches en plein essor grâce aux progrès récents de la chimie et des modes de conditionnement. Il y a aussi Hippolyte Gosse chargé de la médecine légale, Raymond Olivet pour la psychiatrie, Alfred Vaucher pour l'obstétrique.

Mais ce sont les professeurs des sciences médicales de base que le cortège va installer les premiers, puisque après avoir traversé la plaine de Plainpalais et les terrains du jeu de mail, il se rend à l'Ecole de Médecine. L'illustre FriedrichWilhelm Zahn y dispensera ses connaissances étendues en histologie et en anatomo-pathologie, en collaboration avec Sigismond Laskowski pour l'anatomie, et de Maurice Schiff pour la physiologie. On avait d'abord nommé à ce poste, BrownSéquard. Mais, alléché par de meilleures conditions financières, il avait démissionné avant d'entrer en fonctions. Il y eut effectivement quelques grimaces, car le Conseil d'Etat ne s'était pas montré très généreux envers les professeurs. Les conditions étaient particulièrement dures pour ceux de l'Ecole de Médecine qui, ne pratiquant pas, ne pourraient compter sur aucun gain accessoire. Avec huit mille francs par an, on ne vivait pas mieux à l'époque qu'avec quarante mille francs aujourd'hui. Leur traitement allait heureusement s'améliorer rapidement. Bénéficiant de la mauvaise expérience faite avec Brown-Séquard, Schiff se vit déjà octroyer quatre mille francs de mieux !

Mais foin de ces considérations matérielles! Le cortège gravit les marches de l'Ecole de Médecine, où une allocution et la visite des lieux va clore la séance, car l'inauguration de la faculté à l'hôpital sera plus discrète. En effet, la Commission administrative redoute quelque peu les réactions des malades et de la population ${ }^{6}$. Certains n'ont d'ailleurs pas caché leurs craintes, répugnant à l'idée de devenir 
des instruments d'enseignement. A en croire Henri Maunoir, président de la Commission Administrative de l'hôpital pour 1876, tout semble s'être cependant bien passé et cela, grâce, - je cite - «au tact ainsi qu'à l'expérience de MM les professeurs et à la parfaite convenance de MM les étudiants».

L'installation des cliniques a créé, on s'en doute, bien des tracas, un surcroit de travail pour les uns, des sacrifices pour les autres.

Ceux dont l'abnégation mérite certainement le plus grand respect furent Louis Odier et Jean-Louis Prévost, responsables des services ordinaires de chirurgie et de médecine, puisqu'ils perdirent d'un coup près de $80 \%$ de leurs patients au profit des admissions dans les cliniques.

Il semble qu'ils n'aient pas trop regimbé, «comprenant, comme l'écrit Maunoir ${ }^{6}$, que leur intérêt particulier devait plier devant l'intérêt général ». L'année suivante, Louis Odier allait tomber malade et se retirer, laissant sa place à son adjoint, Jaques-Louis Reverdin. Prévost, lui, fut moins à plaindre, car il recevait en contrepartie la chaire de thérapeutique et pharmacologie.

En outre, très rapidement, la fréquentation des services et des cliniques allait retrouver un semblant d'équilibre avec toutefois, toujours un avantage pour les cliniques qui reçurent, en 1877, 15\% de patients de plus que les services. Ces derniers devaient d'ailleurs disparaître en 1882, date à laquelle ils seront absorbés par les universitaires.

Sur le plan pratique, l'entrée de la faculté à l'hôpital ne s'est pas faite sans mal. Il a fallu transformer des locaux et en créer d'autres. Ainsi, la salle de chirurgie du rez-de-chaussée fut convertie en amphithéâtre. Nous verrons plus loin son mode d'utilisation. Le deuxième étage qui servait de grenier, fut totalement aménagé en salles de malades avec salles de bains et salle de traitement. L'ouverture de ce deuxième étage a nécessité l'installation d'un ascenseur, destiné tant au transport des malades qu'à celui des vivres et des effets.

L'arrivée des cliniques servit en outre d'excellent prétexte pour améliorer les conditions d'hygiène de l'hôpital. On introduisit l'eau courante à tous les étages et dans les toilettes. Cette innovation consommant beaucoup d'eau, il fallut se munir de pompes à vapeur pour remplir les réservoirs aménagés dans les combles.

Pour lutter contre la pourriture d'hôpital, on appliqua les préceptes de dispersement dont Florence Nightingale avait si bien démontré l'efficacité lors du conflit de Crimée. Cinq pavillons furent créés dans les jardins et le système allait donner tant de satisfaction qu'en 1877 on en érigea quatre supplémentaires. Notons, en passant, que, depuis l'avènement des antibiotiques, les médecins ont cru pouvoir oublier ces sages précautions et ont laissé construire d'immenses blocs hospitaliers 
qui offrent une promiscuité où prolifèrent, ravis, staphylocoques dorés, pseudomonas et cafards.

L'aération dans les salles fut améliorée par un système de ventilation à hélices actionné par la pompe à vapeur.

Les sols furent recouverts de parquets plus faciles à garder salubres que les dalles de molasse qui s'imprégnaient de pus, de sang ainsi que de toutes sortes d'autres liquides peu recommandables.

On transforma et agrandit la morgue, complément indispensable de tout service universitaire. On acheta aussi une ambulance neuve, car, à l'époque, c'était l'hôpital qui se chargeait du transport des malades.

Il fallut en outre acquérir de la lingerie, des lits, des sommiers, des meubles, des poêles calorifères, des instruments, bref tout un matériel qui allait faire grimper le prix de la journée d'hôpital, au grand désespoir de la commission administrative et du Conseil d'Etat.

Afin d'enrayer les gaspillages, on créa de nouveaux postes, on nomma un économe chargé du contrôle et de l'emploi des fournitures. On créa aussi une pharmacie capable de confectionner elle-même les préparations qu'on achetait auparavant au prix fort dans les officines de la ville.

Mais la réalisation la plus onéreuse fut incontestablement la transformation de l'ancien Hôpital de Plainpalais en Maternité. Chose extraordinaire et qu'jl vaut la peine de relever, le devis ne serait pas dépassé. On croit rêver...

Toutes ces dépenses se firent naturellement avec l'accord du Conseil d'Etat qui avait débloqué un crédit spécial à cet effet. Notons qu'une part importante de cette allocation provint de la succession du duc de Brunswick, ce qui devrait nous permettre de supporter un peu mieux la laideur de son mausolée.

Sur le plan humain, l'introduction de la faculté a sensiblement bouleversé la vie de l'hôpital. Si l'accroissement du corps médical et paramédical apporta une amélioration notable des soins, la loi de Parkinson joua : le travail augmenta proportionnellement au personnel disponible. Les malades souffrirent de surmenage, car leur repos était de plus en plus souvent troublé par des visites qui, de surcroît, duraient davantage. A tout instant, un groupe d'étudiants faisait irruption dans les salles pour examiner tel ou tel cas qui, s'il était spécialement intéressant, se voyait en outre, exposé dans l'amphithéâtre. En contrepartie, l'accroissement des discussions autour de leur maladie devait sinon flatter leur vanité, du moins rassurer les malades sur le souci qu'on prenait d'eux. Ils se sentirent cependant souvent perdre leur qualité d'homme pour devenir progressivement des cas. Leur moral s'en ressentit au point que la direction s'en émut. Pour leur procurer de la distraction et plus de gaieté, on créa des réfectoires où 
ils purent prendre leurs repas en commun, jouer aux cartes et deviser tranquillement.

A l'hôpital, l'enseignement était en grande partie dispensé au lit du malade, lors de la visite du matin et, le soir, à la consultation des policliniques.

Les cours ex cathedra avec ou sans présentation de cas constituèrent une innovation de taille à l'hôpital. C'est l'amphithéâtre du rez-de-chaussée qui servit de salle de cours. Les professeurs de chirurgie y effectuaient aussi des démonstrations opératoires. Après présentation du cas, discussion du diagnostic et de la thérapeutique, on amenait le futur opéré déjà endormi au chloroforme - l'éther purifié ne serait réintroduit qu'à partir de 1877 -, allongé sur une table roulante. De part et d'autre de cette table d'opérations, on disposait une estrade amovible ${ }^{3}$, à trois niveaux, sur laquelle les étudiants prenaient place debout. Comme on opérait peu à l'époque (43 opérations pour les deux services en 1876) ${ }^{6}$, ces séances constituaient un événement dont les étudiants, on s'en doute, étaient friands. Par la suite, le nombre des opérations allait rapidement augmenter pour dépasser 200 en 1880. C'est que, en même temps que la faculté, les pratiques d'avant-garde comme l'antisepsie et les narcoses à l'éther purifié firent leur entrée à l'hôpital. De nombreux cas jusqu'alors considérés comme désespérés se virent offrir avec moins de réticence la solution d'une intervention.

Cependant, comme la nécessité d'opérer vite interdisait de trop longues explications, les étudiants s'entraînaient sur des cadavres, au laboratoire d'anatomie de l'école de médecine.

A l'intérieur des cliniques et des divers services, les patients étaient pris en charge, comme de nos jours, par des internes rétribués nommés annuellement. Ils restaient généralement en poste jusqu'à l'obtention de leur doctorat. Leur nombre variait, car ils cumulaient parfois plusieurs fonctions. En 1876, ils atteignirent à peine la douzaine, en comptant les assistants de l'école de médecine.

Grâce à la présence de ces internes, l'installation des cliniques allait instaurer le règne sacro-saint du dossier. En effet, il devenait impératif que chaque observation puisse servir à la recherche clinique. Dès 1876 , le dossier allait prendre de l'ampleur, y gagnant en précision et en détails. Pourtant, nos aînés surent résister à la papyrophilie morbide qui ronge les médecins modernes. En quelques coups de plume, ils traçaient l'essentiel de l'observation clinique qui, en l'absence de moyens d'investigation poussés, demeurait la pierre angulaire de l'élaboration des diagnostics. A ce sujet, le dossier médical portant le $\mathrm{N}^{0} 1$ dans les archives, est tout-à-fait significatif. Il concerne un magasinier de 22 ans souffrant de maux de tête et de crampes intestinales intermittentes mais fort intenses. Ce jeune homme présentait en outre des taches bleuâtres sur le thorax et un liséré bleuté soulignait 


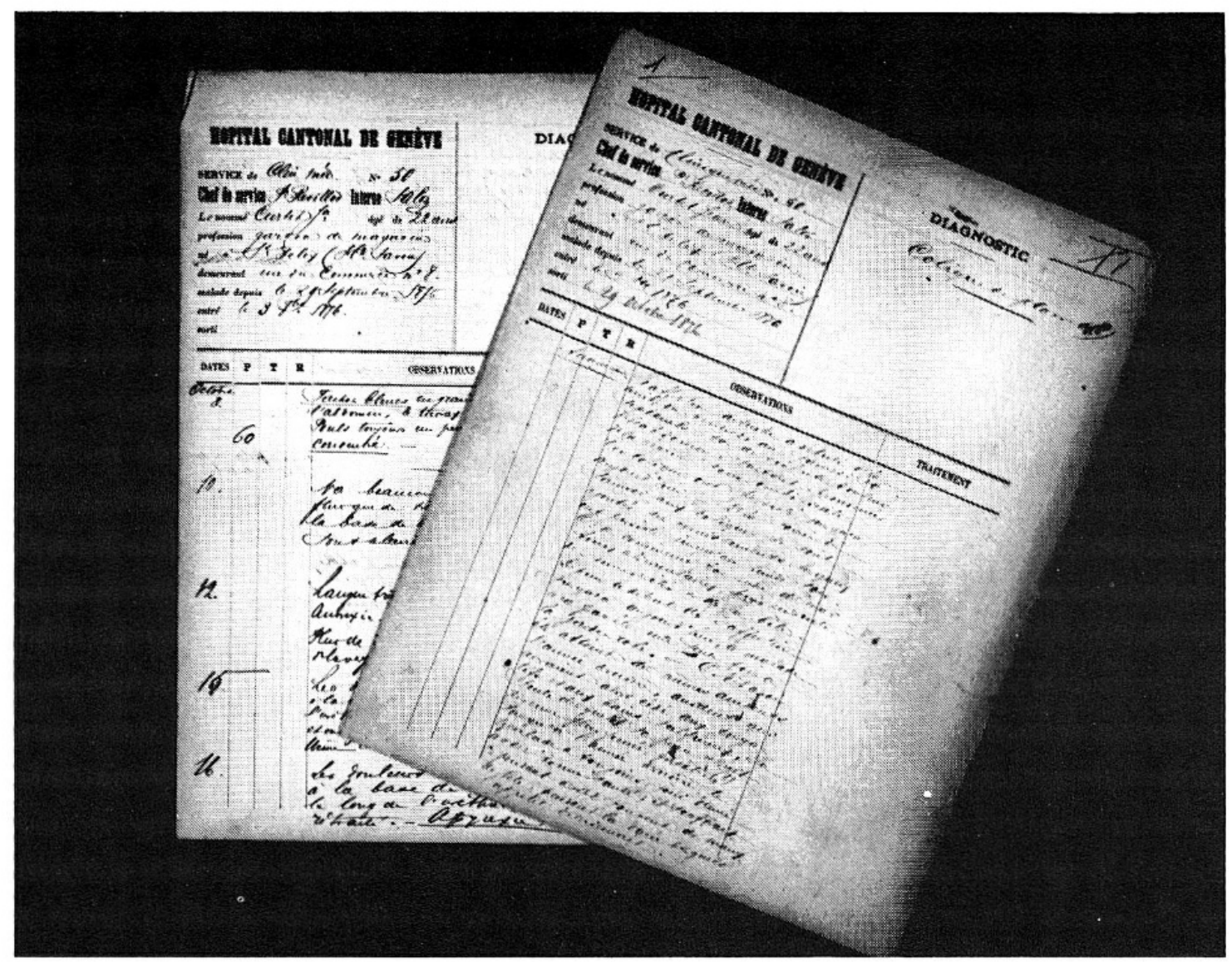

Fig. 1. Le dossier classé sous le chiffre 1

ses gencives. Pour qui connait sa sémiologie, le diagnostic d'intoxication au plomb ou saturnisme ne fait pas de doute. Ce qui est remarquable c'est que, cent ans plus tard, cette observation demeure tout-à-fait utilisable pour celui qui entreprendrait une recherche sur les intoxications au plomb.

Nous voyons donc que, d'emblée, les buts fondamentaux de la faculté sont atteints : soin des malades, enseignement, recherche et études cliniques.

Le plan d'enseignement qu'il serait fastidieux d'énoncer ici, différait étonnamment peu de celui d'aujourd'hui. Cela ne nous surprend pas puisque le sujet de base n'a guère varié : l'homme, la maladie et son traitement. Toutes les branches introduites par la suite demeurent dans le même cadre délimité par la clinique, la pathologie et la thérapeutique.

Le programme des cours, tel qu'il avait été conçu avant l'inauguration de la faculté, allait séduire un nombre respectable d'étudiants, puisque le semestre d'hiver 1876 compta déjà quarante-sept étudiants réguliers, dont quinze Genevois et vingt confédérés, ce qui montre bien l'importance de la faculté sur le plan 
romand. Mais cet attrait allait dépasser d'emblée les frontières, puisque sur les quarante-sept premiers, il n'y eut pas moins de douze étrangers (près de $30 \%$ ) dont deux français, deux grecs, un italien, un brésilien, un polonais et cinq russes. Lorsqu'ils arrivèrent, ils se trouvaient évidemment à des niveaux de formation divers.

Le premier à terminer son cycle d'étude, sanctionné par un examen final et la soutenance de thèse, fut Alfred Vincent, en 1878, déjà.

A la manière américaine actuelle, les interrogations portaient sur toutes les connaissances accumulées depuis le début des études. Mais le nombre réduit des candidats autorisait des formes très détendues. Les examens oraux se passaient sur rendez-vous, au gré des examinateurs et du candidat. La consultation écrite d'un cas clinique illustre bien la confiance détendue qui présidait à ces épreuves. Sans aucune surveillance, le candidat examinait un patient. Il rédigeait ensuite son observation qu'il allait remettre au Professeur ; s'il était absent, il lui envoyait simplement sa copie par la poste!

Avec les examens, la publication des thèses allait concrétiser l'efficacité de la nouvelle institution ${ }^{7}$. Comme c'est sur sa production que l'on juge un organisme, penchons-nous sur les premiers fruits de l'union de la faculté à l'hôpital. La diversité des dix premiers sujets traités témoigne de la vitalité de cette toute jeune faculté, comme des excellentes conditions de recherche fournies par les structures hospitalières et para-hospitalières.

Nous l'avons vu, le travail d'Alfred Vincent porte le numéro un. C'est une étude de la plus haute importance sur les anomalies artérielles, une recherche impressionnante par sa minutie et son ampleur. Malheureusement, comme souvent à l'époque, elle est alourdie de trop nombreuses considérations philosophiques dues au faible développement des sciences connexes qui forçait les auteurs à produire des arguments hypothétiques plus souvent basés sur la morale que sur les faits.

Plus visuel, Charles Roy, dans la thèse numéro deux, résista mieux à cette tendance. Il faut dire que son sujet ne prête guère aux envolées métaphysiques: les effets des purgatifs au niveau de l'intestin. Son travail n'en demeure pas moins un chef-d'œuvre d'expérimentation pharmacologique, surtout les chapitres réservés à l'effet macroscopique de l'administration de purgatifs, laxatifs et drastiques. Ne disposant pas des moyens modernes de la photographie médicale, il produisit des planches d'une grande précision, chaque détail y ayant sa signification.

Du même genre et dirigée par le même patron, la thèse de Kohler, portant le numéro trois, consigne les effets des diurétiques : eau, nitrate de potasse, acétate d'ammoniaque, alcool, digitale. Il y a une mention spéciale pour le curare que 

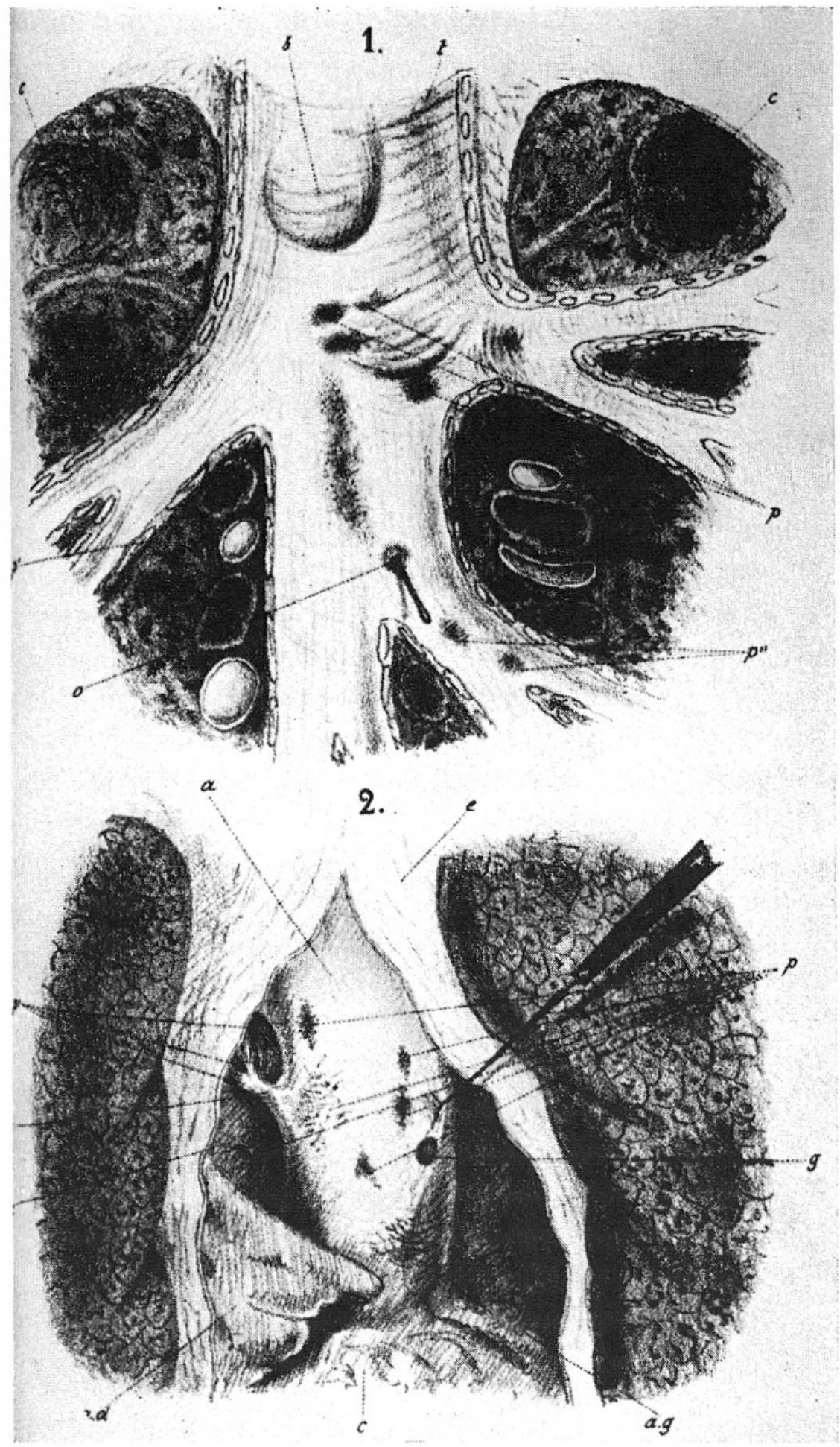

Fig. 2. Complications macroscopiques des adénopathies para-trachéobronchiques. Thèse d'Auguste Eternod 
l'auteur, n'ayant trouvé aucun volontaire, avala,... sans effet diurétique. Son enthousiasme ne lui donna heureusement pas le courage de s'injecter le produit !

La thèse numéro quatre d'Auguste Eternod passe en revue un énorme chapitre de la pathologie : celui des adénopathies inter- et para-trachéobronchiques, avec leurs complications locales et à distance. Là aussi, l'iconographie dut paraître sous la forme de dessins d'une qualité remarquable.

Pour le numéro cinq, Antonin Favre effectua de longues et patientes recherches sur les corpuscules amyloïdes contenus dans l'urine de certains patients, touchant ainsi du doigt le problème très complexe de l'amyloïdose.

Puis, ce furent les études de Charles Saloz sur le phénomène respiratoire de Cheyne-Stokes; celles de Louis Wintzenried sur la brucine, un alcaloïde de la noix vomique moins toxique que la strychnine dans le traitement du tétanos; celle de Henri Martin sur les suppurations métastatiques de la gonorrhée, une pathologie que nous ne connaissons heureusement plus; celles très spécialisées du russe Michaloff sur les métastases de l'enchondrome, une tumeur osseuse assez rare.

La première thèse féminine porte le numéro dix. Nous la devons à Olga Gortinsky qui apporta une contribution de taille à la compréhension de la dégénérescence des nerfs en étudiant la durée de leur excitabilité après leur section.

Arrêtons-nous là, car le bref survol de ces dix premiers travaux démontre amplement l'allant de notre faculté qui, en 1877, dans un hôpital rénové, transformé, allait pouvoir accueillir sans complexe les membres du Congrès International des Sciences Médicales. 


\section{Références et bibliographie}

${ }^{1}$ Bickel, Georges, La faculté de Médecine de Genève, de 1876 à nos jours. Le $70^{\mathrm{e}}$ anniversaire de la fondation de la faculté de Médecine. Allocutions et discours. Genève (Georg \& Cie) 1948.

${ }^{2}$ Eternod, Auguste-C.-F., Université de Genève, Faculté de Médecine 1876-1896, Genève (Georg \& Cie) 1896, et in: Borgeaud, Charles, Histoire de l'Université de Genève, 4 vol. et 2 annexes, Genève (Georg \& Cie) 1934.

${ }^{3}$ Giraud, Charles, La Clinique Chirurgicale dans l'Université de Genève. Historique des Facultés 1896-1914. Genève (Georg \& Cie) 1914.

${ }^{4}$ Julliard, Gustave, Statistiques concernant la Faculté de Médecine, Genève (Impr. Souillier) 1884 .

${ }^{5}$ Roch, Maurice, Libre histoire médicale des cent premières années de l'hôpital cantonal de Genève. In : Centième anniversaire de l'Hôpital Cantonal de Genève, 1856-1956, Genève (Impr. Populaires SA) 1956, p. 39-156.

${ }^{6}$ Rapports de la Commission administrative de l'hôpital cantonal de Genève. 1876 et 1877. Genève (Impr. Charles Pfeffer).

${ }^{7}$ Recueil de thèses de la Faculté, Vol. 1 et 2, Bibliothèque de la Faculté de Médecine, Genève.

\section{Summary}

The faculty, inaugurated on October 23, 1876 in the presence of an imposing professorial group, reached at first trial its basic goals: improvement of hospital services, teaching, research, and clinical studies. The hospital was considerably transformed and improved. The introduction of teaching disturbed its routine, but attracted numerous students. Careful case histories and the enthusiasm of young researchers resulted in considerable and varied scientific activities as witnessed by the 10 first doctoral dissertations.

Dr. Jean de Blonay

17, avenue du Mail

1205 Genève 